\title{
ENCEFALOPATIA DE WERNICKE
}

\section{A PRÓPOSITO DE UM CASO COM SÍNDROME PILÓRICA OBSTRUTIVA}

\author{
HÉLIO ARAÚJO OLIVEIRA * JOSÉ FÁBIO SANTOS LFOPOLDINO* *
}

\begin{abstract}
RESUMO - Os autores apresentam um caso de encefalopatia de Wernicke em um paciente com síndrome de obstrução pilórica por adenocarcinoma gástrico, sem história de alcoolismo e desnutrição. Comentam aspectos fisiológicos da importáncia da tiamina como co-enzima da transcetolase e da piruvato-descarboxilase na rota metabólica para produção de ATP e a possibilidade da existência de interação de fatores genéticos e ambientais no surgimento do quadro clínico. Chamam a atenção para a importância do diagnóstico clínico, que deve ser precoce para que a terapêutica específica com tiamina possa surtir efeito, principalmente em pacientes sem historia prévia de alcoolismo e desnutrição.
\end{abstract}

PALAVRAS-CHAVE: encefalopatia de Wernicke, tiarnina, obstrução pilórica, adenocarcinoma gástrico.

\section{Wernicke's encephalopathy: report of a case with obstructive pyloric syndrome}

SUMMARY - The authors present one case of Wernicke's encephalopathy in a patient with obstructive pyloric syndrome caused by gastric adenocarcinoma, wihout previous history of alcoholism or malnutrition. They comment on the importance of physiological aspects of thiamine as a co-enzyme of transketolase and pyruvatedecarboxilase in the metabolic pathway for ATP production and the possibility of existing an interaction between genetical and environmental factors in the onset the symptoms. They draw attention for the clinical diagnosis, which should be precocious for the therapy with thiamine to be efective, especially in patients without previous history of alcoholism or malnutrition.

KEY WORDS: Wemicke's encephalopathy, thiamine, pyloric obstruction, gastric adenocarcinoma.

A encefalopatia de Wernicke (EW) é enfermidade neurologica potencialmente grave, causada pela deficiência de tiamina, que funciona como co-fator enzimático da rota metabólica na sua fase anaeróbica, sendo portanto fundamental na produção de ATP ${ }^{22}$. A EW ocorre com frequência em pacientes alcoólatras e desnutridos ${ }^{6}$. Nestes casos o diagnóstico torna-se mais fácil; entretanto, outras situações clínicas podem estar associadas à $\mathrm{EW}$, tais como: hiperemese gravídica ${ }^{1,14,16,21,23}$ obstruçāo do trato gastrointestinal alto ${ }^{5.22}$, alimentação venosa prolongada ${ }^{9}$, jejum prolongado ${ }^{3}$, nefropatias crônicas em hemodiálise ${ }^{4}$ e vigência de tratamento quimioterápico ${ }^{1}$. Nestas condições clínicas o diagnóstico tem sido feito tardiamente, em virtude de sempre a $\mathrm{EW}$ ter sido relacionada a alcoolismo e desnutriçāo ${ }^{25.10}$. Este diagnóstico tardio tem contribuido para que grande número de pacientes nāo possa se beneficiar com a terapêutica adequada que, quando instituida tardiamente, apresente resultados catastróficos.

Estudo realizado no Hospital Universitário da Universidade Federal de Sergipe (UFS): *Professor Adjunto do Departamento de Medicina - Clínica Neurológica; **Médico Estagiário da Clínica Neurológica. Aceite: 16janeiro-1995.

Dr. Hélio Araújo Oliveira - Rua Reginaldo Passos Pina 261, Parque dos Coqueiros - 49040-000 Aracajú SE Brasil. 
Neste artigo os autores relatam um caso de EW, em que o paciente apresentava síndrome obstrutiva pilórica, por neoplasia gástrica, sem passado de alcoolismo ou desnutrição, cujo diagnóstico foi feito tardiamente, interferindo na resposta à terapêutica instituida. Aproveitam a oportunidade para fazer revisão dos aspectos etiológicos e fisiopatológicos da doença.

\section{RELATO de CASO}

MANM, 46 anos, masculino, foi internado na Enfermaria de Clínica Médica do Hospital Universitário - UFS, para estadiamento de neoplasia gástrica. A história mostrou que há 4 meses o paciente, que até entāo gozava de boa saúde, passou a apresentar vômitos alimentares tardios, ocasionais, com alimentos parcialmente digeridos e odor fétido, associados a plenitude pós-prandial e pirose. Passados dois meses do início dos sintomas, os vômitos já eram bem frequentes e ocorriam logo após a ingesta de qualquer alimento. Pirose intensa, perda de peso e astenia foram relatados. Foi feito o diagnóstico clínico de síndrome pilórica; realizada esofagogastroduodenoscopia, que revelou estenose pilórica; biópsia demonstrou presença de adenocarcinoma gástrico. Nesta época o paciente já apresentava discretos episódios de lapsos de memória, com amnésia anterógrada e retrógada de forma transitória, com nítido comprometimento da memória de fixaçāo. Ao internar apresentava queixa de diplopia, evidenciava estrabismo convergente bilateral e exacerbação da amnésia, estado apático confusional global com profunda desatenção, indiferença ao meio ambiente e desorientação tempo/espaço. Exame físico: estado geral regular, perda de peso moderada ( $5 \mathrm{~kg}$ em quatro meses); aparelhos e sistemas sem alterações; sinais vitais preservados. Apresentava hipersonia, desatenção e apatia. As pupilas eram mióticas, isocóricas e com resposta lenta a fotoestimulaçāo. Havia estrabismo convergente bilateral, nistagmo na mirada do olhar lateral. Fundo de olho normal. A motricidade, reflexa e voluntária, estava normal. Apresentava ataxia da marcha, necessitando de apoio para locomoção. Não havia alteração da coordenação dos movimentos voluntários. Foram realizados exames complementares: RX de crânio, RX de tórax; cintilografia cerebral; CT de crânio, exames bioquímicos e hematológicos, que não revelaram qualquer anormalidade. Evoluiu na enfermaria passando de um estado de sonolência para o de topor intenso, quando foi transferido para a unidade de terapia intensiva; dois dias após a internação na terapia intensiva estava em coma, sem sinais de localização neurológica e sem sinais de hipertensāo intracraniana. Os sinais vitais estavam mantidos e avaliaçōes hematológicas e bioquímicas foram normais exceto para os hormônios tireoidianos, que estavam discretamente diminuidos, e pelo aparecimento de hiperglicernia com resposta insatisfatória à insulinoterapia. Foi feita a suspeita diagnóstica de EW e de imediato foi iniciada a reposição de tiamina na dose de $100 \mathrm{mg}$ IV/dia. No quarto dia de uso de tiamina, o paciente havia passado do estado de coma para um estado de sonolência, com resposta a solicitaçð̄es verbais, abertura espontânea dos olhos, desaparecimento do nistagmo e do estrabismo, com sinais de nítida interação com o meio ambiente. Apesar da melhora neurológica o paciente apresentou complicação pulmonar, com o aparecimento de processo infeccioso grave, vindo a falecer no vigésmo dia de internação.

\section{COMENTÁRIOS}

A encefalopatia de Wernicke (EW) é doença caracterizada pela deficiência de tiamina no organismo. Foi descrita de forma organizada pela primeira vez em 1881 por Carl Wernicke que, mesmo estando longe de relacionar a patologia à deficiência de tiamina, nem tão pouco à ingesta de álcool, uma vez que o caso apresentado por ele se referia a ingestão suicida de ácido sulfúrico seguida de estenose esofágica, vômitos incoercíveis e morte, relatou os sintomas e sinais clínicos e demonstrou as alteraçōes anatomopatológicas no encéfalo ${ }^{22}$. Nesta época a evolução para o óbito era inevitável e Wernicke denominou a doença de polioencefalite hemorrágica superior aguda ${ }^{22}$. As manifestações clínicas mais comuns da EW compōem uma tríade clássica: distúrbios oculomotores, ataxia cerebelar e estado confusional ${ }^{6,22}$.

As manifestações oculares são as mais frequentemente encontradas e traduzem-se por paresia ou paralisia bilateral do sexto nervo craniano e nistagmo, que pode ser horizontal e/ou vertical. $O$ nistagmo não é espontâneo, surgindo nas tentativas de mirada em direção à zona acometida ${ }^{22}$. Outras manifestações oculares menos frequentes incluem: oftalmoplegia completa, anisocoria, lentidão do reflexo fotomotor direto, miose, edema de papila e hemorragia retiniana ${ }^{6}$. A ataxia cerebelar expressase na postura e na marcha, cuja intensidade dos sintomas pode variar, indo de alterações detectáveis apenas em testes semióticos, até incapacidade completa para deambulação ${ }^{22}$. $\mathrm{O}$ acometimento quase 
exclusivo dos membros inferiores explica-se pelas lesões na porção anterior e superior do vermis cerebelar $^{12,15}$. Quanto às alteraçōes mentais elas podem ter início insidioso e lento, agravando-se posteriormente ou, por outro lado, manifestar-se subitamente através de confusão mental e queda do nível de consciência, podendo chegar ao coma. Uma miscelânea de manifestações pode ocorrer como por exemplo: letargia, desorientação, amnésia anterógrada e retrógrada, alterações da percepção, perda do senso crítico, indiferença ao meio externo e a si mesmo e hipersonia ${ }^{6,22}$. Quando a EW não se reverte em um prazo de 48 a 72 horas após o início dos sintomas, os pacientes passam a apresentar a psicose de Korsakoff (PK), que se expressa por preservação da memória imediata, alteração da anterógrada, alteração parcial da retrógada, falsos reconhecimentos, fabulações. O surgimento da PK é tão frequente nos portadores de EW que muitos preferem denominar o quadro de síndrome de Wernicke-Korsakoff́ ${ }^{6}$. Outras manifestações clínicas menos frequentes incluem hipotensão e hipotermia, resultantes das lesões hipotalâmicas ${ }^{6}$.

Durante muito tempo a EW foi considerada apanágio exclusivo dos alcoólatras de longa data, portadores de desnutrição crônica ${ }^{6,22}$. Isso em parte se explica pela grande incidência da doença nesse grupo de pacientes. Nos seus trabalhos, Adams, citado por Figueira e Laks ${ }^{6}$, relata que dos $0,13 \%$ dos pacientes internados no Hospital Geral de Boston eram alcoólatras e destes $3 \%$ apresentavam EW. Estudos mais recentes fazem também relacão direta entre EW e alcoolismo e por isto talvez é que o diagnóstico não é feito precocemente naqueles pacientes que não têm história pregressa de alcoolismo ${ }^{6.17}$. A partir de estudos neuropatológicos é que ficou constatada a grande incidência de casos de EW não diagnosticados em vida ${ }^{2,10,13}$. Em estudo de quatro anos, Harper ${ }^{9}$ encontrou prevalência de $0,8 \%$ a $2,8 \%$ de EW nas autópsias realizadas. Lana-Peixoto e col. ${ }^{10}$ relataram em trabalho recente o encontro de 36 casos de EW diagnosticados apenas durante a autópsia. Tudo isso se deve ao fato de que muitos dos pacientes de $\mathrm{EW}$ não apresentam sintomatologia típica. Podem aparecer com apenas uma ou duas das manifestações clássicas e também sem qualquer uma delas. Não são raros os casos de morte súbita ou de coma de etiologia inexplicada atribuídos à EW durante a necrópsia. Por outro lado, muitos dos pacientes que apresentam EW nāo se enquadram no perfil de alcoólatras e desnutridos crônicos. Entre 6964 autópsias, realizadas por Lindboe e Loberg ${ }^{13}$, havia 52 casos de EW dentre os quais $12(23 \%)$ não eram alcoólatras. Ebels ${ }^{5}$ encontrou antecedentes de alcoolismo em apenas 4 dos 22 casos de EW por ele autopsiados.

Pacientes apresentando a tríade clássica são raros. Só $16 \%$ dos portadores de EW têm quadro típico $^{10}$. O nistagmo horizontal está presente em $85 \%$ dos pacientes e a paralisia bilateral do sexto nervo craniano em $54 \%$. Craviotto e col. ${ }^{2}$ encontraram alterações oculares em apenas $50 \%$ de 328 casos de EW. A ataxia correspondia a $15 \%$ e $92 \%$ tinham síndrome mental orgânica. As alterações anatomopatológicas incluem: atrofia, espongiose e descoloração dos corpos mamilares, atrofia cerebelar, hemorragias petequiais periventriculares, proliferação glial, hipertrofia endotelial e necrose da bainha de mielina. $\mathrm{O}$ acometimento destas áreas é facultativo e proporcional à intensidade do processo com exceção dos corpos mamilares, atingidos em $100 \%$ dos casos de $\mathrm{EW}^{10,12,13,15}$. A mielinólise pontina central é rara ${ }^{10}$.

O prognóstico dos pacientes com EW depende basicamente da fase da doença em que é feito o diagnóstico e da imediata instituição do tratamento. Apesar de ser entidade já há muito conhecida e a terapêutica consistir na simples reposição de tiamina, muitos pacientes ainda morrem sem diagnóstico em vida ou evoluem para a psicose de Korsakoff, alguns necessitando de institucionalização permanente. Por outro lado, a reversão completa do quadro pode ser conseguida com o diagnóstico e terapêutica precoces. A mortalidade da EW varia de 10 a $20 \%$; $80 \%$ dos pacientes evoluem para PK e apenas $20 \%$ se recuperam completamente, só que a longo prazo (meses e até anos) ${ }^{6}$. A morte se dá frequentemente por pneumonia, tuberculose pulmonar, septicemia e insuficiência hepática ${ }^{6,22}$.

A deficiência de tiamina é a grande responsável pelo surgimento da EW. Qualquer condição que deplete as reservas orgânicas, principalmente a hepática, pode desencadear a doença. A partir 
dessa premissa concluí-se que não só o alcoolismo ou a desnutrição crônica provocam EW. Nos últimos anos tem crescido o registro de casos com EW sem antecedentes de alcoolismo. Wood e col. ${ }^{23}$ relataram três casos de EW resultante de hiperemese gravídica. Lavin e col." ${ }^{1,}$ também observaram EW em duas pacientes com hiperemese gravídica, submetidas a alimentação venosa prolongada. Da mesma forma, Montane e col. ${ }^{14}$ relataram quadro clínico de $\mathrm{EW}$ em duas pacientes com hiperemese gravídica; nestas pacientes o tempo de duração dos vômitos variou entre 5 a 10 semanas e a perda de peso entre 2 a 16 quilos. Devathasan e $\mathrm{Koh}^{3}$ relataram um caso de $\mathrm{EW}$ associado a jejum prolongado, cujos sintomas apareceram no final de um mês de iniciado o jejum. Handler $e$ Perkin $^{8}$, em um dos seus casos, associaram EW a anorexia nervosa. Harper ${ }^{9}$ relata três casos de EW e alimentação venosa prolongada. Neste mesmo trabalho revê nove casos da literatura; destes, seis faleceram e a variação de tempo referente ao início dos sintomas após a admissão foi de 4 a 80 dias; cinco apresentaram coma e todos faleceram, seis não exibiram qualquer dos sinais clássicos da $\mathrm{EW}$. Soffer e col. ${ }^{20}$ observaram EW em um caso de AIDS por eles autopsiado. Além da síndrome consuptiva, o paciente apresentava amnesia e demência. A partir desta observação, postularam o uso de tiamina parenteral em pacientes aidéticos com síndrome amnésica e/ou demência. $\mathrm{A}$ EW também foi encontrada em pacientes submetidos a hemodiálise por nefropatia crônica ; também, em pacientes, principalmente crianças, em tratamento poliquimioterápico para leucemia aguda linfoblástica ${ }^{1}$.

A tiamina é de extrema importância para o perfeito funcionamento da rota metabólica. Age como co-enzima da transcetolase e da piruvato-descarboxilase, enzimas intermediárias na degradação do piruvato em Acetil-Co-A. A ausência de tiamina bloqueia a entrada do piruvato na fase aeróbica da rota metabólica gerando dois efeitos: desvio da via aeróbica para anaeróbica, onde o piruvato é transformado em lactato, e diminuição da produção de energia (ATP), que passa a ser produzida apenas pela via glicolítica. Resulta daí a acentuada produção de ácido lático no organismo e a deficiência da produção de ATP. Além disso, a transcetolase auxilia a síntese de ácidos graxos, componentes fundamentais da mielina. Não está devidamente esclarecido por que há predileção das lesões pelos corpos mamilares, paredes dos ventrículos e vermis cerebelar. Alguns autores sugerem que as lesões ocorrem nas vias que contêm monoaminas, pois o principal metabólito da noradrenalina (3-metoxi-4-hidroxifenilglicol) encontra-se dimunuído no sistema nervoso central de pacientes com EW. Outro dado é que a clonidina, uma alfa-2-adrenérgico, melhora o distúrbio de memória nestes pacientes $^{6,18,22}$.

Apesar da grande lógica existente nos conhecimentos até agora adquiridos sobre EW, muitos detalhes ainda necessitam ser esclarecidos. Exemplo disto é o fato de que nem todo paciente possuidor de condições propícias para desencadear EW o fazem. A quantidade de casos de EW em países como a Austrália e Brasil, onde há um grande número de alcoólatras e desnutridos, deveria ser bem maior do que se registra ${ }^{7}$. Estas observações levaram alguns autores a racionar sobre a possibilidade de existir uma predisposição individual, para o surgimento da $\mathrm{EW}^{18}$. Além dos distúrbios metabólicos e neurofisiológicos já comprovados, como a redução da atividade da transcetolase e piruvatodescarboxilase e do comprometimento do sistema nervoso central serotoninérgico, existe a possibilidade da presença de anormalidade genéticas, como alterações das propriedades cinéticas da transcetolase, condição esta já inerente ao paciente antes da depleção de tiamina. A partir daí, há a hipótese de ser a EW o resultado da deficiência de tiamina em um paciente com prévia reduçāo da afinidade da transcetolase por esta vitamina. Seria uma interação entre fatores genéticos e ambientais ${ }^{21}$.

A tiamina está presente na maioria dos alimentos, principalmente nos cereais. Nos tecidos humanos transforma-se em sua forma ativa, a tiamina pirofosfato, que servirá de cofator enzimático para produçāo de ATP. Quanto ao seu estoque, o hepatócito funciona como principal local de armazenamento. Ziporin e col. ${ }^{24}$, mostraram que, uma vez cessada a ingesta de tiamina, as reservas orgânicas esgotam-se em 18 dias. Condiçōes como doença hepática, ingesta excessiva de carbohidratos ou infusão venosa de grandes quantidades de glicose, não associada à tiamina, podem acelerar essa depleção. Atenção especial deve ser dada ao uso de glicose hipertônica sem a devida reposição de 
tiamina, prática comum principalmente em alcoólatras ou pacientes em coma de etiologia não definida, pois pode vir a desencadear EW ou piorar um quadro já instalado ${ }^{24}$. O percentual de pacientes com EW que tiveram o coma como manifestação principal tem crescido bastante. A alta incidência de estados comatosos não é um fato novo, uma vez que a patologia da $\mathrm{EW}$, compromete o sistema reticular ativador ascendente (SRAA), responsável pela manutenção do estado de vigília, em decorrência de sua projeção difusa sobre o córtex cerebral ${ }^{15}$. Lana-Peixoto e cols ${ }^{10}$, estudando 36 casos de EW autopsiados constataram que 11(30,6\%) haviam desenvolvido coma, todos sem diagnóstico em vida. Muitos estudos têm sido feitos e a incidência de casos de coma associado a $\mathrm{EW}$ varia de 10 a $80 \%$. Nos trabalhos de Harper ${ }^{9}$, a EW só é diagnosticada clinicamente em $20 \%$ dos casos. Isso levou vários autores a preconizarem o uso de tiamina de forma sistemática nos pacientes em coma sem etiologia definida ${ }^{4.7,9}$.

No que se refere ao diagnóstico laboratorial, os exames rotineiros na maioria das vezes não se encontram alterados ou, se estão, não sugerem o diagnóstico, pois são inespecíficos. Exemplo destes são as provas de função hepática, os eletrólitos séricos ou a gasometria arterial. Alguns pacientes podem apresentar um quadro hiperglicêmico, confundido com diabetes mellitus, mas resistente ao uso de insulina. Dosagens séricas específicas, mas muito sofisticadas, incluem: medida do piruvato plasmático após administração de glicose, medida da transcetolase eritrocitária, ativação da flavina e outros ${ }^{\Downarrow}$. Por não contar com estes exames em nosso meio é que o diagnóstico clínico tem sido cada vez mais valorizado. Os exames covencionais por imagem (RX simples de crânio, cintilografia cerebral, tomografia axial computadorizada) também nāo conseguem esclarecer o diagnóstico, pois as alterações anatômicas são muito pequenas, quando não microscópicas. A ressonância nuclear magnética, entretanto, devido ao seu alto poder de resolução, pode demonstrar alteraçōes dos corpos mamilares, muito antes mesmo do aparecimento do quadro clínico característico da $\mathrm{EW}^{19}$. $\mathrm{O}$ eletroencefalograma revela lentificação difusa do traçado, sem especificidade.

O tratamento baseia-se na reposição de tiamina, inicialmente por via parenteral (IM ou IV), na dose de $100 \mathrm{mg}$ por dia, com o objetivo de reverter os sintomas oculares, principalmente a oftalmoplegia e as alteraçōes de consiciência ${ }^{7}$. Às vezes são necessárias doses de até $1000 \mathrm{mg}$ nas primeiras 12 horas de internação $0^{6}$. Naqueles pacientes submetidos a alimentação venosa prolongada, doses de 50 a $500 \mathrm{mg}$ de tiamina diárias são necessárias para prevenir $\mathrm{EW}^{3}$. Pacientes que receberam $10 \mathrm{mg}$ diariamente desenvolveram assim mesmo a síndrome ${ }^{8}$. O requerimento diário de tiamina depende da quantidade de calorias incorporadas ao organismo e é da ordem de $0,4 \mathrm{mg} / 1000 \mathrm{Kcal} /$ $\mathrm{dia}^{24}$.

No caso relatado, houve grande dificuldade no estabelecimento do diagnóstico. O quadro de adenocarcinoma gástrico associado a quadro de paralisia bilateral do nervo abducente, ataxia e síndrome amnésica levou a suspeita inicial de processo metastático intracraniano. A partir dos resultados dos exames complementares que descartaram a possibilidade de processo metastático, $o$ diagnóstico de EW foi considerado, levando em conta dados da história e exame físico do paciente. Com relação à história clínica, o paciente apresentava um quadro emético persistente, amenizado pelo uso de metoclopramida e dieta pastosa, mas capaz de reduzir seu peso em cerca de cinco quilos. A evolução protraida e a presença da tríade clássica, estado confusional, ataxia cerebelar e alterações oculomotoras, foram os primeiros indícios da patologia. Não havia sinais clínicos ou tomográficos de hipertensão intracraniana; não foram encontradas alterações vasculares, degenerativas ou anatômicas de qualquer espécie que pudesse justificar o quadro clínico. Os exames complementares normais serviram para descartar a possibilidade da presença de endocrinopatias. Vários casos já foram publicados em que os exames complementares não mostraram qualquer alteração ${ }^{21}$. $O$ estudo do LCR permitiu afastar doença infecciosa aguda ou sub-aguda, inclusive infecção fúngica. $O$ surgimento de quadro de hiperglicemia resistente à insulinoterapia reforçou a impressão diagnóstica de EW. A evolução do caso após o uso da tiamina confirmou a suspeita. 
A dificuldade em diagnosticar EW em pacientes não alcoólatras e com bom estado geral se deve ao baixo índice de suspeita para essa patologia neste grupo. Até o momento, muitos profissionais da área da saúde têm relacionado a EW a alcoolismo ou desnutrição grave. Resulta, assim, que grande número de pacientes vem a falecer sem diagnóstico de EW ainda em vida ou vêm a ter sequela mental, pelo diagnóstico tardio. Um diagnóstico precoce associado a uma terapêutica adequada, o uso de tiamina em casos de pacientes em coma com etiologia indefinida e precaução na utilização de glicose hipertônica, ou alimentação venosa prolongada, são grandes armas para mudar o prognóstico nefasto da EW.

\section{REFERENCIAS}

1. Brack W, Cristen HJ, Lakomek H, Hanefeld F, Friends RL. Wernicke's encephalopathy in a child with acute lymphoblastic leukemia treated with polychemoterapy. Clin Neuropathol 1991, 10:134-136.

2. Craviotto H, Korein J, Silbur J. Wernicke's encephalopathy: a clinical and pathological study of 28 autopsied cases. Arch Neurol 1961, 4:510-519.

3. Devathasan G, Koh C. Wernicke's encephalopathy in prolonged fasting. Lancet 1982, 13:1108-1109.

4. Descambes E, Dessibourg CR, Felloy G. Acute encephalopathy due to thiamine deficiency (Wernicke's encephalopathy) in a cronic dialyzed patient: a case report. Clin Nephrol 1991, 35:171-175.

5. Ebels EJ. Underlying illness in Wernicke's encephalopathy: analysis of possible causes of under diagnosis. Eur Neurol 1974, 12:226-228.

6. Figueira ICV, Laks J. Encefalopatia de Wernicke. J Bras Psiquiatr 1988, 37:33-38.

7. Fischer AJ, Yellowless PM. Prevention of the Wemicke-Korsakoff syndrome in Australia; a cost-benefit analysis. Med J Aust 1989, 150:331-333.

8. Handler CE, Perkin GD. Anorexia nervosa and Wernicke's encephạlopathy: an underdiagnosed association. Lancet 1982, 2:771-772.

9. Harper CG. Sudden unexpected death and Wernicke's encephalopathy: a complication of prolonged intravenous feeding. Aust N Z J Med 1980, 10:230-235.

10. Lana-Peixoto MA, Santos EC. Pittella JE. Coma and death in unrecognized Wernicke's encephalopathy. Arq Neuropsiquiatr 1992, 50:329-333.

11. Lavin PSM, Smith D, Kori SH, Ellenberger C Jr. Wernicke's encephalopathy: a predictable complication of hyperemesis gravidarum. Obst Gynecol 1983, 62:13-15.

12. Lindboe CF, Erichsen AA. Atrophy and sponginess of the mammillary bodies with neuronal sparing: not only inactive Wernicke's encephalopathy. APMIS 1989, 97:667-670.

13. Lindboe CF, Loberg EM. Wernicke's encephalopathy in non-alcoholics: an autopsy study. J Neurol Sci 1989, 90:125-129.

14. Montane V, Imbert Y, Dupont E, Pelegrini JL, Leng B. Encephalopatie de Gayet-Wernicke par vomissements gravidiques. Press Med 1990, 19:83-84.

15. Nakada T, Knight RT. Alcohol and the central nervous system. Med Clin N Am 1984, 63:121-131.

16. Nightingale S, Heath PD, Bates D, Bassan SL. Wernicke's encephalopathy in hyperemesis gravidarum. Postgrad Med J 1982, 58:558-559.

17. Olvera R, Juan E, Moreno MEV, Rosas JS, Ochoa EV. Neuropathologic lesions associated with chronic alcoholism. Rev Invest Clin 1986, 38:375-381.

18. Schenker S, Henderson GI, Hoyumpa AM, McCandless DW. Hepatic and Wernicke's encephalopathies: current conceps of pathogenesis. Am J Clin Nutr 1985, 33:2714-2726.

19. Shogry ME, Curner JT. Mammillary body enhancement on MR as the only sign of acute Wernicke's encephalopathy. Am J Neuroradiol 1994, 15:172-174.

20. Soffer F, Zinkin H, Alkan C, Bergines VM. Wernicke's encephalopathy in acquired imune deficiency syndrome (AIDS): a case report. Clin Neuropathol 1989, 8:192-194.

21. Turner S, Daniels L, Greer S. Wernicke's encephalopathy in a 18-year-old-woman. Br J Psychiatry 1989, 154:261-262.

22. Victor $M$, Adams R. Doenças carenciais do sistema nervoso. In Harrisson TH. Medicina interna Ed 10. Rio de Janeiro: Guanabara - Koogan 1984, Vol 2, p 2345-2351.

23. Wood P, Murray A, Sinha B, Gadley M, Goldsmith HJ. Wernicke's encephalopathy induced by heperemesis gravidarum: case report. Br J Obstet Gynecol 1983, 90:583-586.

24. Ziporin ZZ, Nunes WT, Powell RC. Thiamine requirement in the adult human mensured by urinary excretion of thiamine metabolites. J Nutr 1965, 85:297-301. 